\title{
Ensemble of Segmentation Methods for Detecting Breast Cancer
}

\author{
Shashank A R ${ }^{1}$, M.Veena ${ }^{2}$ \\ M Tech, Dept of Computer Science and Engineering, PESCE, Mandya ${ }^{1}$ \\ Assistant Professor, Dept of Computer Science and Engineering, PESCE, Mandya ${ }^{2}$
}

\begin{abstract}
Breast cancer could be a considerable prime cause that affects the lives of women's and results in death all round the world. Consistent with applied math information from the WCR, the proportion of breast tumors in cancers diagnosed is up to thirtieth, leading to fifteenth death because of cancer worldwide. The goal of the primary stage is to boost the image mistreatment totally different pre-processing filters that removes the noise and highlights the tumor region. Within the next stage, tumor is extracted from increased pictures mistreatment projected segmentation techniques classified as delicate, benign and malignant.
\end{abstract}

Keywords: Pre-processing, filters, enhance, segmentation

\section{INTRODUCTION}

According to the yank Cancer Society, regarding one in eight U.S. girls can develop invasive carcinoma over the course of her time period. Nearly all twenty five of carcinoma deaths occur in girls diagnosed between forty to forty nine years. Early detection and treatment are presently the sole suggests that tried to cut back carcinoma connected mortality rates. Early detection of carcinoma still remains as open drawback within the analysis community. A digital Breast Tomosynthesis is employed for the detection of carcinoma. this is often conjointly known as as three-dimensional diagnostic technique, it uses the low radiation $\mathrm{x}$-ray system and three-dimensional pictures of the breast are created by pc reconstructions. Digital Breast Tomosynthesis overcome the restrictions of the traditional roentgenogram. In standard roentgenogram overlapping of the breast tissue results in loss of the data. however in DBT the X-ray tube moves in arc over the every breast wherever it gets multiple $\mathrm{X}$-ray photos and from that we will get a lot of info and conjointly it permits the detection of tiny tumors that helps within the more treatment choices. DBT can find the tiny carcinoma that's hidden within the standard roentgenogram.DBT conjointly avoid extra screening and also diagnostic test. In standard roentgenogram it takes solely the pictures from 2 directions that are from prime to bottom and aspect to side and contrariwise.

\section{EXISTING SYSTEM}

The existing systems are Breast rumination (BSE), mammogram, clinical breast examination, microwave imaging and ultrasound. Breast self examination: it's a screening technique that involves girls herself viewing her breast and feeling every breast for attainable lumps or swelling. the difficulty with breast rumination is that it's not tried to save lots of the life and it is not habitually counseled by health authorities. Mammogram: it always takes 2 x-ray of every breast from completely different angles prime to bottom and aspect to side and contrariwise. a number of the problems are the compression of breast that's needed throughout a X ray are often uncomfortable. The compression can cause the overlapping of breast tissues, thus overlapping of the breast tissue isn't visible within the mammographic pictures. Clinical breast examination: it's a physical examination that's done by the tending supplier. the difficulty is that it's to be performed solely by the health care supplier well trained within the technique. Microwave imaging: it's a promising technique for early stage detection of carcinoma. Ultrasound: it's AN imaging check that sends high frequency sound waves through breast and converts that into pictures on viewing screen. It places a sound emitting probe on the breast to conduct the check. it's not used on its own as screening check for carcinoma, rather it's to enhance different screening tests. Since the ultrasound pictures are of low intensity it's not appropriate for early detection.

\section{PROPOSED SYSTEM}

Here we've taken DBT pictures as input and at first perform preprocessing for an input image here we tend to are applying opera hat transformation, Otsu thresholding and adaptative thresholding the most task in preprocessing is to boost the image by removing the noise while not destroying its original properties. Then in segmentation, apply Otsu thresholding on adaptative and opera hat transformation then fuse each Otsu thresholding on adaptive and Otsu 
Vol. 8, Issue 4, April 2019

thresholding on top hat. Image segmentation divides the region into non-overlapping areas. It segments the tumour in white region and remainder of the world are going to be reborn into black. the most advantage of fusion technique is it combines with completely different thresholding results in order that the noise are going to be cleared and improved distinction and cut back grey level image to a binary image so that up background exploit and image improvement will be done.

\section{SYSTEM ARCHITECTURE}

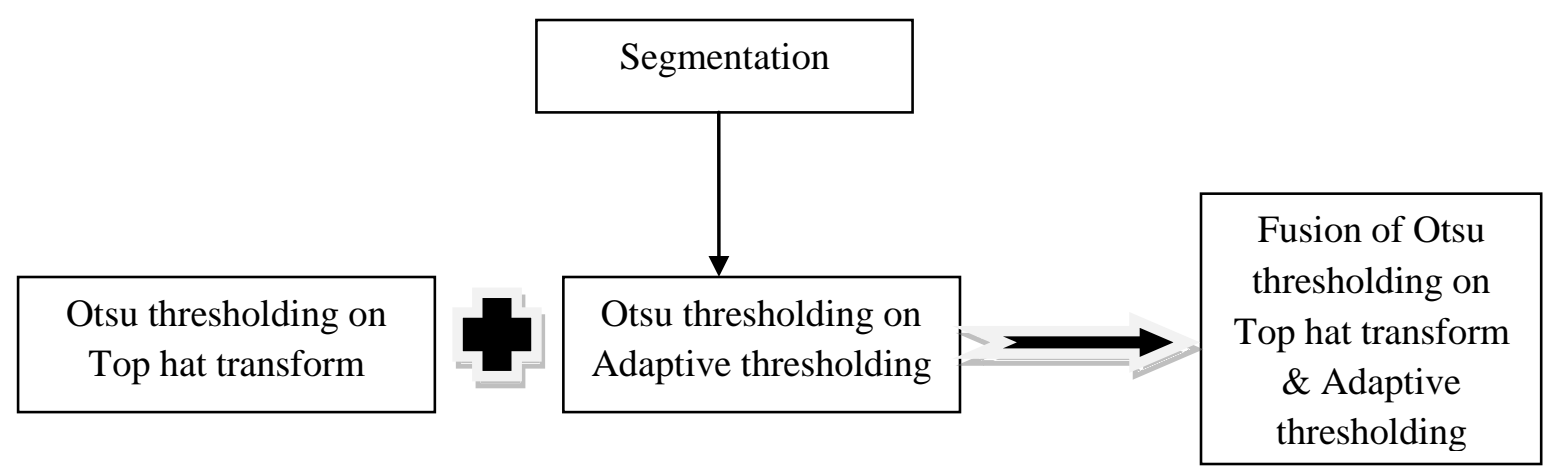

Fig1: Fusion technique

\section{METHODOLOGY}

i. $\quad$ Pre-processing

The purpose of the pre-processing module is to scale back or eliminate a number of the variations within the noninheritable image. It normalizes and increased the tomo image to boost the popularity performance of the system. The pre-processing is crucial because the strength of the system greatly depends thereon. a collection of processes is employed in knowledge preparation and filtering to get rid of the noise. Associate in Nursing intelligent implementation is employed to form it prepared for later analysis later with the aim to modify psychological feature of the image and its content while not human facilitate. The techniques used at this section are completely different in line with the character of data required to be extracted from the image. There are several functions in MATLAB that facilitate in developing image process systems like (imread, rgb2gray, imadjust, im2bw, bwareaopen, bwboundaries, impixel etc.) in order that the image will be pre-processed for the segmentation section.

\section{ii. Segmentation}

Image segmentation is one among the first steps in image analysis for object identification. the most aim is to acknowledge homogenous regions at intervals a picture as distinct and happiness to completely different objects. The segmentation method will be supported finding the utmost homogeneity in grey levels at intervals the regions known. There are many problems associated with image segmentation that need careful review. one among the common issues encountered in image segmentation is selecting an acceptable approach for uninflected completely different objects from the background. Image improvement techniques get to boost the visual look of a picture. They emphasize the salient options of the first image and alter the task of image segmentation. The type of operator chosen includes a direct impact on the standard of the resultant image. it's expected that a perfect operator can enhance the boundary variations between the objects and their background

Here, we've an inclination to are applying Otsu thresholding on every top-hat work and adaptive thresholding. Otsu thresholding technique might be a classification-based technique that searches for the brink that minimizes the intraclass variance, made public as a weighted total of variances of the two classes. Otsu thresholding technique corresponds to the linear discriminate criteria that as- sums that the image consists of only object (foreground) and background, and additionally the non uniformity and form of the background is ignored, Otsu set the brink so on try to minimize the overlapping of the class distributions. The goal is to look out the brink worth with the minimum entropy for total of foreground and background. Otsu technique determines the brink worth supported the maths information of the image where for a specific threshold price t the variance of clusters T0 and T1 could also be computed. the most effective threshold worth is calculated by minimizing the whole of the weighted cluster variances, where the weights are the possibility of the assorted groups.

Given: $p(i)$ because the bar graph possibilities of the discovered grey worth $i=1, \ldots, 1$

$$
p(i)=\text { number }\{(r, c) \mid \operatorname{image}(r, c)=i\}
$$



image, severally.

$w_{b}(t), \mu_{b}(t)$, and $\sigma_{b}^{2}(t)$ as the weight, mean, and variance of class T0 with intensity value from 0 to $\mathrm{t}$, respectively.

$w_{f}(t), \mu_{f}(t)$, and $\sigma_{f}^{2}(t)$ as the weight, mean, and variance of class T1 with intensity value from $\mathrm{t}+1$ to 1 , respectively.

$\sigma_{w}^{2}$ as the weighed sum of group variances.

The best threshold worth $t^{*}$ is that the value with the minimum inside category variance. The inside category variance defines as following:

$\sigma_{w}^{2}=w_{b}(t) * \sigma_{b}^{2}(t)+w_{f}(t) * \sigma_{f}^{2}(t)$

Where:

$w_{b}(t)=\sum_{i=1}^{t} p(i)$

$w_{f}(t)=\sum_{i=t+1}^{l} p(i)$

$\mu_{b}(t)=\sum_{i=1}^{t} i * p(i) / w_{b}(t)$

$\mu_{f}(t)=\sum_{i=t+1}^{l} i * p(i) / w_{f}(t)$

$\sigma_{b}{ }^{2}(t)=\sum_{i=1}^{t}\left(i=\mu_{b}(t)\right)^{2} * \mathrm{p}(\mathrm{i}) / w_{b}(t)$

$\sigma_{f}^{2}(t)=\sum_{i=t+1}^{l}\left(i=\sigma_{f}^{2}(t)\right)^{2} * \mathrm{p}(\mathrm{i}) / w_{f}(t)$

In mathematical morphology and digital image method, top-hat make over is associate operation that extracts very little parts and details from given footage. There exist two kinds of top-hat rework: The white top-hat remodel is made public as a result of the excellence between the input image and its gap by some structuring element; The black top-hat transform is outlined dually because the distinction between the closing and additionally the input image. Top-hat transforms are used for various image method tasks, like feature extraction, background effort, image improvement. And adaptive thresholding, thresholding can be a straightforward and effective means that of partitioning an image into a foreground and background. This image analysis technique can be a range of image segmentation that isolates objects by dynamical grayscale footage into binary pictures. Image thresholding is barely in footage with high levels of distinction. Then applying Otsu thresholding on each high hat and adaptative thresholding here we have a tendency to get 2 totally different results by fusing those two results we get a stronger output.

This division into components is commonly supported the characteristics of the pixels within the image. for instance, a method to search out regions in a picture is to seem for abrupt discontinuities in pel values, which generally indicate edges. These edges will outline regions. alternative strategies divide the image into regions supported color values or texture. Edges in pictures are regions with terribly high distinction in intensity of pixels; detection of edges reduces the quantity of information, filters useless info and preserves vital structural details. There is a awfully sizable quantity of edge detection operators out there, each designed to be sensitive to sure types of edges. Variables involved among the selection of an edge detection operator embrace Edge orientation, Noise surroundings and Edge structure

\section{iii. $\quad$ Feature Extraction and classification}

Gabor filter is in addition remarked as linear filter that's used for texture analysis, that suggests that it primarily analyzes whether or not or not there are any specific frequency content among the image in specific directions in an exceedingly \} very localized region around the purpose or region of analysis. And classify test-image exploitation KNN whether or not or not it's mild, benign or tumour.

\section{CONCLUSION}

As per survey early detection of carcinoma continues to be a challenge. there's scope for improvement. Every modalities have their own limitations, so fusion of data retrieved from totally different modalities could also be useful to enhance the accuracy of early detection of carcinoma. The techniques developed among the four stages like preprocessing, segmentation, feature extraction and selection, classifications are summarized. completely totally different fusion techniques are studied, and thus the long run developments and trends are also investigated. This paper explored the evaluations of preprocessing and segmentation techniques exploitation ensemble primarily based approaches. The experimentation is conducted within the UPMC Breast pictorial representation and FFDM assortment info of mammograms. 


\title{
International Journal of Advanced Research in Computer and Communication Engineering
}

\author{
Vol. 8, Issue 4, April 2019
}

\section{REFERENCES}

[1]. John H. Hipwell, Thomy Mertzanidou, Kalnisha Naidoo, Norman Williams, Sarah Pinder, and David J. Hawkes, “Automated Classification of Breast Cancer Stroma Maturity From Histological Images” IEEE TRANSACTIONS ON BIOMEDICAL ENGINEERING, VOL. 64, NO. 10, OCTOBER 2017.

[2]. Jose A. Martinez Lorenzo, Richard Obermeier, Fernando Quivira, Carey M. Rappaport Richard Moore, Daniel B. Kopans "Fusing DigitalBreast-Tomosynthesis and Nearfield-Radar-Imaging Information for a Breast Cancer Detection Algorithm" C2013 IEEE.

[3]. Sangeetha R Dr. Srikanta Murthy K “A Novel Approach for Detection of Breast Cancer at an early stage by Identification of Breast Asymmetry and Microcalcification cancer cells using Digital Image Processing techniques” 2017 2nd International Conference for Convergence in Technology.

[4]. S.Sasikala, M.Ezhilarasi," Fusion of Two View Binary Patterns to Improve the Performance of Breast Cancer Diagnosis" International Conference on Communication and Signal Processing, April 6-8, 2017, India.

[5]. R.Meena Prakash, K. Bhuvaneshwari, M. Divya, K. Jamuna Sri, A.Sulaiha Begum, "Segmentation of thermal infrared breast images using KMeans, FCM and EM algorithms for breast cancer detection” 2017 International Conference on Innovations in Information, Embedded and Communication Systems.

[6]. Renaud Morin, Bj”orn Eiben, Luc Bidaut, John Hipwell, Andrew Evans, David J. Hawkes, "3D ULTRASOUND SIMULATION BASED ON A BIOMECHANICAL MODEL OF PRONE MRI IN BREAST CANCER IMAGING” C2015 IEEE.

[7]. Salar Razavi *, G“okhan Hatipo־glu†, H“ulya Yalc, ın, "Automatically Diagnosing HER2 Amplification Status for Breast Cancer Patients Using Large FISH Images” @2017 IEEE

[8]. Lifan Xu, Baosheng Wang, Zhansong Lin, Xiong Wang, “A Novel Monitoring Technique for Breast Cancer Hyperthermia Using Thermoacoustic Imaging" 2018 IEEE.

[9]. S. Di Meo, G. Matrone, M. Pasian, "High-Resolution mm-Wave Imaging Techniques and Systems for Breast Cancer Detection" IEEE MTT-S International Microwave Workshop Series on Advanced Materials and Processes (IMWS-AMP 2017), 20-22 September 2017, Pavia, Italy.

[10]. Sergey Vesnin, Igor Goryanin, "Modern microwave thermometry for breast cancer” ICIIBMS 2017, Track3: Bioinformatics. Medical Imaging, and Neuroscience, Okinawa, Japan. 